Lexis Vol. XLV (2) 2021: 623-657

\title{
De azada a trillo: los nombres de instrumentos agrícolas en el COSER y en el CORPES XXI. Repercusiones lexicográficas"
}

\author{
Jacinto González Cobas \\ https://orcid.org/0000-0003-3974-398X \\ Universidad Autónoma de Madrid \\ jacinto.gonzalez@uam.es
}

\begin{abstract}
Resumen
Los especialistas coinciden en que se han producido cambios de gran calado en la manera de planificar y confeccionar los diccionarios. Uno de ellos es la búsqueda de canales a partir de los cuales intensificar la colaboración entre la lingüística teórica y la lexicografía; sin embargo, no siempre se concretan las fórmulas para que tal postulado no sea una mera declaración de intenciones. Los corpus son una herramienta para que esa relación tenga lugar y son una referencia para el establecimiento de modelos definitorios aplicables a grupos homogéneos de palabras, en nuestro caso, los nombres que designan instrumentos agrícolas, que han recibido un tratamiento lexicográfico harto heterogéneo en la última edición del Diccionario de la lengua española (DLE) de la RAE.

Palabras clave: COSER, CORPES XXI, nombres de instrumentos agrícolas, modelos de definición
\end{abstract}

"Este trabajo se ha realizado en el marco del proyecto de investigación PGC2018095077-B-C42, dirigido por Inés Fernández-Ordóñez y Ana Serradilla Castaño.

https://doi.org/10.18800/lexis.202102.004 
From azada to trillo: The nouns designating agricultural tools in the COSER and the CORPES XXI. Lexicographic Implications

\begin{abstract}
Experts agree that there have been significant changes in the way dictionaries are planned and produced. One of them is the search for channels from which the collaboration between theoretical linguistics and lexicography can be intensified; nevertheless, the formulae needed to prevent such postulate from being a mere statement of intent are not always adequately fixed. Corpora are tools which enable that relationship, and they are a reference for the establishment of definition models applicable to homogeneous groups of words, in our case, the nouns designating agricultural tools, which have received a very heterogeneous lexicographic treatment in the last edition of the Diccionario de la lengua española (DLE) by RAE. Keywords: COSER, CORPES XXI, nouns designating agricultural tools, definition models
\end{abstract}

\title{
1. INTRODUCCIÓN
}

En los últimos años se ha extendido la idea de que los avances alcanzados dentro del terreno de la lingüística teórica han de aprovecharse para mejorar ostensiblemente la confección de los diccionarios tanto en lo referente a su proceso de elaboración como a los resultados derivados del mismo. Se trata de que los datos de índole lingüística guíen etapas cruciales en la configuración de los diccionarios, desde la fase inicial de diseño y planificación hasta otras más avanzadas, como la redacción de las definiciones o su actualización. Al fin y al cabo, la relación entre lingüística teórica y aplicada ha de ser estrecha y, sin duda, la primera puede ayudar sobremanera a que la segunda se desarrolle más y mejor. No obstante, en el caso de la lexicografía no siempre se concretan adecuadamente las fórmulas que sustenten dicha relación y que hagan patente la manera en que la aplicación de determinada información lingüística puede ayudar a que el diccionario gane en coherencia y rigor, a pesar de que hacerlo 
supone la adopción de esquemas metodológicos muy provechosos para abordar las cada vez más complejas tareas a las que se enfrentan los lexicógrafos.

En este artículo nos proponemos justamente presentar líneas de conexión entre los datos recabados por las corrientes lingüísticas actuales y la lexicografía, y lo haremos tomando como referencia los nombres de instrumentos agrícolas, los cuales conforman un grupo de sustantivos que no ha recibido un tratamiento demasiado sistemático, al menos si nos fijamos en las definiciones incluidas al respecto en la última edición del diccionario académico. Nos serviremos de dos corpus (el COSER ${ }^{1}$ y el CORPES XXI²) para constatar el modo como se ve reflejada en el uso la información proporcionada por la lingüística teórica y, asimismo, comprobar si todos los datos aportados por esta última tienen repercusiones de igual calado en el ámbito lexicográfico.

\section{SOBRE LA RELACIÓN ENTRE LA LINGÜÍSTICA TEÓRICA Y LA LEXICOGRAFÍA}

Dos de los aspectos en los que la lingüística teórica puede nutrir a la lexicografía es en lo relativo a la parcelación del léxico y a la aplicación de modelos de definición a los grupos de vocablos resultantes de aquella. Hacemos esta afirmación porque el hecho de que los diccionarios (al menos, los publicados en papel) presenten sus lemas por orden alfabético no tiene como consecuencia necesariamente que sus respectivas definiciones hayan de llevarse a cabo de manera aislada y arbitraria, y mucho menos si se trata de diccionarios digitales, que ofrecen, en principio, un espectro de búsquedas y funcionalidades mucho mayor que el de los diccionarios tradicionales. De hecho, esta es una premisa nada aconsejable que trasluce la falta de un método claro de trabajo y de actuación y que genera no pocos problemas, entre ellos, una carencia pronunciada de sistematicidad. 
Parte de la bibliografía se ha hecho eco de estas cuestiones y ha puesto de relieve la necesidad de relacionar lingüística y lexicografía, así como las ventajas que acarrea esta forma de actuación. Haensch, Wolf, Ettinger y Werner aluden a este asunto en los siguientes términos:

En muchos casos, la influencia de la lingüística moderna ha contribuido a mejorar los métodos de elaboración de los diccionarios: mejor distinción de unidades léxicas pertenecientes a subconjuntos, descripción más exacta de las unidades léxicas, análisis componencial, incorporación de los elementos de formación de palabras, desarrollo sintagmático de las entradas (construcción y régimen, fraseología, sintagmas lexicalizados), aplicación de principios de selección más objetivos que la sola competencia lingüística del lexicógrafo o el aprovechamiento de diccionarios existentes: frecuencia, distribución y disponibilidad (1982: 535-536).

En una línea similar cabe situar a Pascual, quien aboga por aplicar los resultados de la teoría lingüística y de la técnica lexicográfica por medio del principio de coherencia. Así, sugiere organizar las palabras en distintos grupos, “acudiendo primero al criterio categorial, y al semántico después” (1996: 198).

Gross (1998), por su parte, convencido también de que las unidades léxicas no pueden ser aprehendidas como entidades aisladas, señala que es imprescindible describir todas las propiedades lingüísticas de tales unidades para ser capaz de reconocer y generar el conjunto de usos que ha de reflejarse en los diccionarios. Se trata de un postulado que se enmarca en su teoría de clases de objetos, las cuales son entendidas como clases semánticas construidas a partir de criterios sintácticos, integradas en un marco relacional que se propone como alternativa a ciertas taxonomías más basadas en aspectos intuitivos que en datos lingüísticos concretos ${ }^{3}$.

\footnotetext{
3 Algunas de estas clases de objetos pueden ser argumentos: HUMANO / ANIMAL / VEGETAL / INANIMADO CONCRETO (medios de transporte, alimentos, cosméticos...) / LOCATIVO (lugares, países, regiones...) / TEMPORAL (meses, días, horas...); o predicados: HUMANO (hijo, padre, vecino...) / INANIMADO ABSTRACTO (acciones, estados, cualidades) / ACONTECIMIENTO (accidentes, ruidos, catástrofes, ceremonias, fiestas...).
} 
En este escenario cabe situar a Herrero Ingelmo, para quien "las unidades léxicas no pueden ser consideradas como entidades aisladas, suficientes en sí mismas: deben ser definidas en función de su empleo en el marco de la oración en la que se insertan" (2004: 453). Este autor analiza concretamente la gramática de una subclase de fenómenos acústicos (las manifestaciones colectivas de adhesión o rechazo), que pueden aparecer como acciones o bien acontecimientos.

Apresjan (2000), por su parte, en un libro cuyo título es harto significativo (Systematic Lexicography), nomina tipos lexicográficos a los grupos de palabras que comparten propiedades y comportamiento lingüístico y que, por ello, deben ser definidos de manera homogénea en los diccionarios. Ello no excluye, por supuesto, que no haya propiedades no compartidas entre las unidades léxicas que también haya que poner de manifiesto.

Batiukova también defiende la integración lingüística-lexicografía y alude al hecho de que la primera se ha aproximado mucho a la lexicografía por ser esta última "una materia que trata muy de cerca el componente léxico, y viceversa, por el progresivo reconocimiento por parte de los lexicógrafos de la necesidad de incorporar los avances de la lingüística para describir adecuadamente el nivel léxico" (2009: 231). La autora aboga por la aplicación de los principios del lexicón generativo ${ }^{4}$ a la descripción lexicográfica del léxico y pone como ejemplo de ello la forma de operar en SIMPLE (Semantic Information for Multipurpose Plurilingual Lexicons), EuroWordNet y la Ontología Semántica de Brandeis (Brandeis Semantic Ontology, BSO) 5 .

En González Cobas (2010) se propone parcelar el léxico a partir de criterios lingüísticos como fórmula para extraer datos relevantes

\footnotetext{
4 Véanse Pustejovsky (1995) y Pustejovsky y Batiukova (2019).

5 Según informa Batiukova (2009), SIMPLE es un proyecto financiado por el Programa de Ingeniería Lingüística de la Comisión Europea que persigue el desarrollo de lexicones semánticos de amplio alcance; EuroWordNet es una base de datos multilingüe (holandés, italiano, español, alemán, francés, checo y estonio); y BSO es un proyecto del Departamento de Informática de Brandeis University que busca la creación de recursos léxicos basados en la teoría del lexicón generativo.
} 
de cara a la confección de modelos definitorios para determinados grupos de palabras e incluso de un vocabulario definidor ${ }^{6}$, pues, como se pone de manifiesto en González Cobas (2016: 136), "la existencia de un $\mathrm{V}$ [ocabulario] $\mathrm{D}$ [efinidor] permite relacionar lingüística y lexicografía de un modo más patente que por medio de otros procedimientos".

Bosque (2014) enfoca este asunto teniendo en cuenta las potencialidades que ofrece el diccionario digital y, por ello, en la ponencia inaugural ${ }^{7}$ del Simposio Internacional El futuro de los diccionarios en la era digital ${ }^{8}$ alude a la necesidad de que los nuevos diccionarios (digitales) contengan las múltiples conexiones gramaticales y léxicas que los diccionarios en papel no pueden plasmar como consecuencia de su linealidad, así como informaciones que en la actualidad figuran dispersas en diccionarios de variado tipo. Battaner (2014) suscribe estas ideas y considera que debe aprovecharse el hecho de que actualmente el léxico ocupe un lugar prominente entre las preocupaciones de la lingüística y que se esté tratando de agrupar las palabras en clases para darles un tratamiento homogéneo. La académica cataloga como reto de los diccionarios futuros la confluencia entre léxico y gramática.

Soler i Bou (2014), por su parte, señala que "el diccionario no puede ser visto como un fin en sí mismo, sino que el único fin debe ser la descripción del léxico guiada y fundamentada por la lingüística" Así, explica, en su condición de coordinador general del Diccionari Descriptiu de la Llengua Catalana, que "la descripción lingüística, y no la estrictamente lexicográfica, del léxico catalán fue el objetivo fundamental que determinó, de un modo más claro, el diseño del proyecto y de su plan de ejecución". Se trataba de "crear una estructura de datos lingüísticos articulada en un modelo relacional”.

\footnotetext{
6 Por vocabulario definidor se entiende, como es sabido, un léxico controlado a partir del cual se codifican las definiciones.

7 El título de su ponencia no puede ser más significativo: Ideas para conectar palabras. 8 Simposio celebrado del 5 al 7 de noviembre de 2014 en la Real Academia Española.

9 No se proporciona el número de páginas de las citas porque estas proceden de un simposio publicado en Internet: https://tinyurl.com/y32vgucz (Consultado el 01 de junio de 2020).
} 
Soler i Bou enmarca estas consideraciones en el cambio de paradigma iniciado en las últimas décadas del siglo pasado, propiciado por factores como las aportaciones realizadas desde la lingüística, sobre todo en lo referido a la descripción del léxico y al análisis del significado; los avances habidos en la metalexicografía; la aparición de la lexicografía computacional; la generalización del uso de corpus de referencia; así como el desarrollo de la informática. La relación lingüística-lexicografía es, pues, uno de los aspectos determinantes en la renovación metodológica que se está produciendo desde hace años en la elaboración de los diccionarios y su relevancia no se limita a las acciones emprendidas en etapas intermedias o finales, sino que alcanza, en algunos casos, la propia fase de planificación de proyectos lexicográficos muy relevantes.

Uno de esos proyectos (con seguridad, el de mayor envergadura de todo el ámbito hispánico) es el Diccionario bistórico de la lengua española (DHLE), de cuya dirección se ha encargado hasta 2020 el académico José Antonio Pascual ${ }^{10}$ y cuya consecución es uno de los objetivos prioritarios de la Real Academia Española. En la página web de esta institución se explican algunos pormenores de este proyecto ${ }^{11}$ :

El Diccionario histórico de la lengua española (DHLE) es un diccionario nativo digital que persigue describir en su integridad (en el eje diatópico, diastrático y cronológico) la historia del léxico de la lengua española. Una característica definitoria de este repertorio radica en su voluntad de analizar la historia del léxico en una perspectiva relacional, atendiendo a los vínculos etimológicos, morfológicos y semánticos que se establecen entre las palabras. El DHLE ha sido concebido desde sus orígenes como una base de datos léxica electrónica (y diacrónica), lo que permite elaborar sus artículos de acuerdo con un criterio de organización del trabajo por campos semánticos (o voces relacionadas por su significado) y familias léxicas.

10 El director de la Real Academia Española, Santiago Muñoz Machado, ha asumido provisionalmente la dirección de este proyecto.

11 Tomado de https://www.rae.es/recursos/diccionarios/nuevo-diccionario-historico (Consultado el 23 de septiembre de 2021). 
Estas cuestiones son detalladas por Campos Souto, coordinadora del citado diccionario, quien precisa que

En el $N D H E^{12}$, en consonancia con lo que ocurre con la mayor parte de los diccionarios actuales de nueva planta, incluso los que no son electrónicos, se ha optado, pues, por estudiar las palabras relacionándolas de distintas maneras: hemos empezado por seleccionar aquellas que pertenecen a un mismo universo, como es el de las armas, el de los instrumentos musicales, el de las medidas y el de las enfermedades. Partiendo de esos grupos, se ha continuado por las relaciones morfológicas de cada palabra; se ha continuado con sus relaciones semánticas; y se ha llegado a establecer otras relaciones, como es el caso de las gráficas y de las sintácticas (en cuya formalización se está trabajando ahora intensamente) (2015: 82).

Asimismo, Salas Quesada y Torres Morcillo, que también trabajan en la elaboración del DHLE, explican, partiendo de una concepción relacional del léxico de una lengua, que la redacción se realiza por grupos de palabras relacionadas semánticamente (no por orden alfabético ni de manera arbitraria) y que, por ejemplo,

se parte de cada vocablo seleccionado para redactar algunas de las palabras relacionadas con él por sinonimia, así como sus familias o subfamilias léxicas, con lo que de esta aproximación obtenemos una visión de conjunto enriquecedora para el estudio de las voces en particular y de los fenómenos que se muestran recurrentes entre los sinónimos o entre las palabras de una misma familia (2015: 18).

También habrá datos relativos a otras relaciones semánticas, como posibles hiperónimos, hipónimos, holónimos, merónimos, antónimos, complementarios, inversos y reversos.

En realidad, lo que se pretende conseguir es una especie de diccionario total (Menéndez Pidal 1953, idea retomada por Pascual 2015: 7), por la gran cantidad de información que se pondrá a disposición de un abanico muy amplio de destinatarios (el perfil de los mismos se ensancha considerablemente) y en cuya recogida,

12 NDHE (Nuevo diccionario histórico del español) es la anterior denominación del DHLE. 
selección y codificación la lingüística teórica habrá tenido y está teniendo ya un indiscutible protagonismo. Se conseguirá, en línea con los deseos de Bosque (2014) para los diccionarios digitales, integrar en un solo diccionario datos que normalmente es necesario consultar en fuentes diversas.

En cualquier caso, y para terminar este apartado, es importante señalar que la aplicación de los postulados de la lingüística teórica a la lexicografía no siempre está exenta de problemas. Esto es algo de lo que ya se percataron Haensch y otros:

En otros casos, hemos visto que la lexicografía no puede llevar a la práctica todos los postulados formulados por los lingüistas, o sólo por las limitaciones materiales ya mencionadas, sino también porque el diccionario, que se dirige a un público no iniciado en la lingüística (el 99,9\%), no puede exigir del usuario que se familiarice con todo un complejo sistema de reglas de uso y términos complejos (1982: 536).

Batiukova también se refirió a este punto posteriormente:

Las relaciones entre la lingüística teórica y la lexicografía no pueden caracterizarse en términos de un trasvase directo del conocimiento teórico a los diccionarios. Una de las razones de ello, como apunta Geeraerts $(1989)^{13}$, es que los principios de la lexicografía y los de la lingüística difieren notablemente a consecuencia de su orientación: básicamente aplicada en el primer caso y fundamentalmente teórica en el segundo. Por eso la lexicografía aplica los principios lingüísticos de modo indirecto, en muchos casos difícilmente perceptible y no siempre coherente desde el punto de vista de la teoría, pero plenamente justificado por razones prácticas (como las necesidades del tipo concreto de usuarios a los que va dirigida la obra y su nivel de familiaridad con las nociones lingüísticas).

13 Geeraerts, Dirk (1989): "Principles of Monolingual Lexicography”, en Wörterbücher: ein internationales Handbuch zur Lexikographie. Eds. Franz Josef Hausmann, Gerold Ungeheuer, Hugo Steger, Herbert Ernst Wiegand y Armin Burkhard. Berlin: De Gruyter, vol. 1, 287-296. 
Otra índole de razones que obstaculizan la realización de los postulados lingüísticos en la práctica lexicográfica tiene que ver con el hecho de que los modelos teóricos más influyentes se caracterizan por una constante evolución, que requiere cautela por parte de los que quieran aplicarlos: se deben seleccionar los planteamientos más sólidos, los que han pasado la prueba del tiempo (2009: 232).

\section{Propiedades lingüísticas de los NOMbres de instrumentos}

Señalábamos anteriormente que vincular la lingüística con la lexicografía había dado lugar, junto a otros factores, a que autores como Soler i Bou (2014) hayan hablado incluso de un cambio de paradigma para referirse a una nueva manera de idear, planificar y hacer diccionarios. Nos planteamos ahora cómo se plasma esta relación en el caso de los sustantivos que designan instrumentos, grupo de palabras que ha recibido en el Diccionario de la lengua española (DLE) de la RAE un tratamiento bastante heterogéneo si nos atenemos a la información que contienen los diversos lemas y a la forma en que esta es codificada (González Cobas 2009, 2011, 2016). Teniendo en cuenta que "la sistematicidad en la lexicografía se interpreta como señal de fiabilidad en la obra” (DeCesaris y Bernal 2005: 464), buscamos fórmulas y propuestas de orden lingüístico que ayuden a eliminar la falta de regularidad.

En una publicación de $2010^{14}$ nos referíamos a algunos de los aspectos caracterizadores de los nombres de instrumentos, los cuales sintetizamos a continuación:

1. Son no predicativos, es decir, no presentan un esquema o estructura argumental, sino que forman parte de él.

2. Son contables (pueden ir precedidos de cuantificadores numerales; no pueden funcionar como CD o atributo si no van precedidos de un determinante; no pueden ir precedidos

14 González Cobas, Jacinto (2010): “Caracterización lingüística de los nombres de instrumentos de medida. Repercusiones lexicográficas”. Revista de Lexicografía. XVI, 39-58. 
de las construcciones pseudopartitivas algo de o un poco de; etc. $)^{15}$.

3. Pertenecen a la categoría de objetos fabricados y, dentro de esta, a los físicos no icónicos.

De estos tres aspectos nos interesa especialmente el último, lo cual no significa que el resto de ellos no tenga también relevancia en términos lexicográficos ${ }^{16}$.

El binomio natural / fabricado es analizado para el francés por Flaux y Van de Velde (2000) y está basado en la existencia o no de un proceso de fabricación en lo relativo al referente y en las repercusiones lingüísticas que ello tiene. Resulta obvio señalar que en palabras como barco, guitarra o teléfono dicho proceso sí existe, frente a lo que sucede con vocablos como lago, mar o montaña. Por otro lado, si algo se fabrica se hace para que ese algo tenga determinada función, y debe haber algún agente que se encargue de dicha fabricación o simplemente de su uso. En ese sentido, puede afirmarse que se trata de nombres télicos (del griego telos, 'final') y agentivos.

Estas son, de manera resumida, las consecuencias lingüísticas que lo anterior acarrea o, mejor, los datos que justifican que se haya introducido tal distinción:

1. Los nombres de objetos fabricados admiten la adjunción de construcciones como sirve para que ponen de manifiesto su telicidad (un teléfono sirve para comunicarse con otras personas), frente a lo que sucede con los naturales (no télicos), que no permiten tal adjunción (?Una montaña sirve para...), al menos en contextos no metafóricos.

\footnotetext{
15 Véanse Bosque (1999) y RAE y ASALE (2009) para obtener más información a propósito de esta cuestión.

16 Sirvan de muestra las premisas dictadas por García Meseguer, según las cuales para definir nombres contables han de utilizarse como sustantivos de apoyo nombres contables, y para definir nombres de materia han de emplearse como sustantivos de apoyo nombres de materia (2008: 32-33).
} 
2. Los nombres de objetos fabricados (agentivos) admiten la adjunción de construcciones del tipo becho/construido/fabricado/creado/inventado por (El barco fue construido por los italianos; El nuevo prototipo de teléfono móvil ha sido ideado por los japoneses), situación contraria a lo que sucede con los de objetos naturales (?El río ha sido construido por... / ? Esta montaña fue creada por... $\left.{ }^{17}\right)$.

3. Los nombres de objetos fabricados admiten complementos de materia (mesa de madera, instrumento de metal), situación que no es posible en los naturales ("río de agua, "árbol de madera), salvo si están modificados: río de agua dulce, árbol de madera blanca. Parece que en estos últimos la materia se percibe como algo inherente, y a ello se debe la necesidad de introducir algún modificador que aporte un rasgo diferencial.

4. Los nombres de objetos fabricados admiten adjetivos que aluden a su buen o mal funcionamiento o a cualidades relacionadas con su función (televisión estropeada, instrumento muy preciso, compás roto, cama cómoda...), adjunción que no es posible con los nombres de objetos naturales.

Los sustantivos de objetos fabricados, además, pueden subclasificarse en físicos y no físicos. Los segundos (llamados idealidades concretas por Flaux y Van de Velde 2000) están constituidos por un pequeño grupo de nombres (soneto, terceto, poema, sonata, fuga, etc.) que muestran como comportamiento diferenciado, respecto de los primeros, el hecho de que no admiten modificadores relacionados con materia (?poema de papel) o tamaño (?soneto grande).

Si descendemos aún más en la clasificación, los físicos pueden subdividirse, a su vez, en icónicos y no icónicos. Entre los primeros cabe incluir nombres como foto, retrato o cuadro, y son los únicos sustantivos de objetos fabricados que no participan de la idea de función y que son, además, predicativos; los no icónicos, por su parte, son argumentales e incluyen nombres de variadas clases

17 Nótese que un contexto de índole religiosa podría favorecer la adjunción de creado por en ejemplos como Las montañas fueron creadas por Dios. 
semánticas: recipientes, todo tipo de instrumentos, edificios, cosméticos, medios de transporte, etc.; asimismo, entablan con cierta facilidad relaciones semánticas de hiperonimia-hiponimia y holonimia-meronimia. Esto significa que, desde un punto de vista lexicográfico, podrían acogerse con cierta facilidad a las definiciones hiperonímicas, es decir, a aquellas que están encabezadas por un hiperónimo al que se añaden restricciones (diferencia específica) para distinguir un determinado lema de sus cohipónimos (Bosque 1982).

Desde una óptica que combina el modelo del lexicón generativo y el de las funciones léxicas de la teoría sentido-texto, Barrios Rodríguez y De Miguel Aparicio (2018) también parten de la distinción entre nombres de tipo artificial o funcional (artefactos) y nombres de tipo natural establecida por Pustejovsky (1995) en el marco del lexicón generativo:

Los primeros designan objetos creados por el hombre, artefactos destinados a un fin, como tarta ( $>$ 'para ser comida') o bicicleta (> 'para montar en ella'). En la definición de los segundos no se codifica información sobre finalidad, aunque en ciertos contextos puede predicarse un uso habitual de su referente; así, patata designa un objeto que 'se come a menudo' y caballo alude a un típico 'animal de montura', pero sus referentes siguen siendo patata y caballo aunque no se les dé ningún uso (Barrios Rodríguez y De Miguel Aparicio 2018: 139-140).

Una consecuencia sintáctica de esta distinción es, por ejemplo, que los nombres de objetos naturales no pueden ir acompañados de ningún verbo que exprese la idea de causar existencia (como hacer), frente a lo que sucede con los nombres de artefactos, en los que tal combinatoria no genera ningún problema: hacer una tarta.

Dentro de los artefactos, las citadas autoras distinguen entre artefactos puros (asociados al rasgo de finalidad y quale télico directo ${ }^{18}$ ),

18 “[...] podemos definir los nombres con Q[uale] T[élico] directo como aquellos que designan un objeto que es el núcleo del complemento directo del evento o actividad denotada por el verbo implicado por el QT. Así, la novela es el complemento directo de leer, y la tarta es el complemento directo de comer" (Barrios Rodríguez y De Miguel Aparicio 2018: 127). 
instrumentos (con rasgo de función y quale télico indirecto ${ }^{19}$ ) y objetos auxiliares (con rasgo de finalidad y quale télico de implicación $\left.{ }^{20}\right)$. Los primeros quedan ejemplificados por nombres como novela, tarta, examen o tratamiento; los segundos, por cuchillo, pincel, avión o jabón; y los terceros, por gafas o escritorio.

La distinción aludida se basa en la aplicación de una serie de pruebas ante las que estos tres grupos de nombres presentan comportamientos distintos:

1. La combinación con el adjetivo fácil: no se interpreta igual novela fácil ('de ser escrita' / 'de ser leída') que un cuchillo fácil ('de ser fabricado’ / *de ser usado') o unas gafas fáciles ('de ser fabricadas' / *de ser usadas').

2. La adjunción del adjetivo antiguo: tampoco se interpretan igual una antigua novela ('novela escrita hace años') que un antiguo cuchillo ('no corta o no se usa ya para cortar') o antiguas gafas ('no se usan ya para ver mejor').

19 "Por su parte, los nombres con QT indirecto son aquellos que designan un objeto cuyo uso tiene un efecto sobre otros objetos; el nombre que designa al objeto se materializa como núcleo del sujeto (este cuchillo corta bien la carne) o forma parte de un complemento preposicional (el carnicero cortó la carne con este cuchillo)" (Barrios Rodríguez y De Miguel Aparicio 2018: 127).

20 "Los nombres del tipo gafas y escritorio se consideran dotados de un quale télico de implicación (Engagement Telic Quale, Im y Lee, 2013). El objeto denotado por un nombre con un QT de implicación interviene para facilitar una determinada actividad, pero ni la lleva a cabo (como los nombres de instrumento cuchillo y pincel, con QT indirecto) ni se ve afectado por el evento (como los nombres de artefacto novela y tarta, con QT directo). Su comportamiento sintáctico difiere tanto del de los nombres de instrumento como del de los de artefacto. Por un lado, no admiten la alternancia entre sujeto y complemento preposicional característica de los nombres instrumentales: alguien ve con las gafas / *las gafas ven; alguien escribe en el escritorio / "el escritorio escribe. Por otro lado, a diferencia de lo que ocurre con los nombres de artefacto, cuando se combinan con empezar solo desencadenan la lectura agentiva: así, he empezado \{las gafas / el escritorio\} solo puede entenderse como 'he empezado a fabricar...' y nunca como 'he empezado a hacer con \{las gafas / el escritorio\} aquello para lo que están destinados (ver / escribir), en lo que coinciden con los nombres de instrumento" (Barrios Rodríguez y De Miguel Aparicio 2018: 127-128). 
3. La modificación del nombre por medio de un adjetivo valorativo (bueno, excelente, pésimo...): es notoria la diferencia entre un cuchillo excelente ('corta muy bien'), una novela excelente ('muy bien escrita' / 'agradable de leer') y unas gafas excelentes ('de buenos materiales') (Barrios Rodríguez y De Miguel Aparicio 2018: 140-141).

Lo interesante del caso es que los sustantivos que designan instrumentos constituyen un grupo delimitado desde un punto de vista lingüístico, tal y como lo demuestran las diversas pruebas a las que han sido sometidos por diferentes autores. Todo ello hace pensar que pueden compartir al menos ciertos aspectos de un mismo modelo de definición ${ }^{21}$, puesto que admiten la adjunción de iguales tipos de construcciones o de unidades léxicas, y porque en ellos el rasgo [función] se perfila como fundamental. Este no es un asunto menor, pues poder aplicar patrones definitorios similares a grupos de palabras emparentados lingüísticamente a partir de cierto criterio contribuye a confeccionar diccionarios rigurosos, coherentes y precisos.

Sin duda, todas estas acciones requieren no poco tiempo, pero hacer de la fase de planificación del diccionario una etapa reflexiva y de recogida de datos de variada índole puede ahorrar más adelante mucho tiempo en la elaboración de las definiciones y en las sucesivas actualizaciones a las que están sujetas los diccionarios. Por otro lado, las repercusiones de emplear en todo momento un método de trabajo bien articulado y con unos objetivos muy definidos son muy positivas para los usuarios por el indiscutible reflejo que tienen en el producto final: "Remember that there is an inverse correlation between the time it takes you to write a definition, and the time it takes the user to process it: the more effort we put into this task as lexicographers, the easier we make life for our users" (Atkins y Rundell 2008: 452).

\footnotetext{
21 Téngase en cuenta que hay instrumentos de tipo muy heterogéneo: musicales, de medida, agrícolas, de tortura, astronómicos, etc.
} 
Por ello, resulta significativo que esta forma de actuación se haya puesto en práctica en diccionarios en los que la inteligibilidad de las definiciones es especialmente importante. Nos referimos a los diccionarios (monolingües) de español como lengua extranjera, en los que los destinatarios son, en principio, usuarios que no dominan completamente el idioma en el que dichos diccionarios están redactados. En este sentido, es relevante lo que afirma Maldonado González sobre el Diccionario de español para extranjeros (2002), a propósito de las definiciones incluidas en él: "Fueron redactadas según unos modelos tipo, lo que daba una gran coherencia interna al cuerpo del diccionario (véanse, por ejemplo, las unidades de medida, los instrumentos musicales, los cargos y las profesiones, etc.)" (2012: 165).

Tampoco parece casual que en un proyecto tan relevante como el Diccionario histórico de la lengua española (DHLE) se estén elaborando las definiciones conforme a ciertos modelos, como queda demostrado por la consulta y comparación de diez nombres de instrumentos de medida, en donde se corrobora fácilmente, además, que se ha optado por la configuración de un vocabulario definidor ${ }^{22}$ :

acetímetro. 1. s. m. Quím. Instrumento que sirve para medir la cantidad de ácido acético contenida en un líquido y, en particular, en el vinagre.

alcoholímetro. 2. s. m. Instrumento que sirve para medir la cantidad de alcohol presente en el aire espirado por una persona.

anemómetro. 1. s. m. Instrumento que sirve para medir la velocidad o la fuerza del viento.

barómetro. 1. s. m. Instrumento que sirve para medir la presión atmosférica.

electrómetro. s. m. Fís. Instrumento que sirve para medir la cantidad de electricidad que tiene cualquier cuerpo.

\footnotetext{
22 El subrayado en las definiciones no es nuestro, sino que figura tal cual en el $D H L E$, al igual que los colores utilizados. Además, en las definiciones de esclerómetro y odómetro no hay palabras subrayadas porque así las hemos encontrado en el citado diccionario, en lo que parece más bien un error que una intencionalidad de actuar de manera diferente con esos lemas respecto de los otros.
} 
esclerómetro. 1. s. m. Miner. Instrumento que sirve para medir la dureza de los minerales o de materiales de construcción.

higrómetro. 1. s. m. Instrumento que sirve para medir la humedad atmosférica.

odómetro. 1. s. m. Instrumento que sirve para medir el número de pasos dados por una persona o de vueltas efectuadas por una rueda $\mathrm{e}$, indirectamente, la distancia recorrida.

taquímetro. 1. s. m. Instrumento que sirve para medir la velocidad de rotación de la máquina en la que generalmente va instalado, expresándola, por lo común, en número de vueltas por unidad de tiempo.

termómetro. 1. s. m. (1689-2017) Instrumento que sirve para medir la temperatura.

Obsérvese la importancia que se da a la función y al hiperónimo, pues ambos aparecen resaltados tipográficamente en el propio diccionario, en consonancia con la información aportada por la lingüística teórica respecto de los nombres que designan instrumentos.

En cualquier caso, creemos que la conveniencia de parcelar el léxico es incuestionable, si bien no negamos que puedan existir fórmulas alternativas a la que aquí se ha presentado. Podría optarse por acudir a clasificaciones de tipo temático, aunque ello tendría como resultado, por ejemplo, que partitura, metrónomo, intervalo, batuta o diapasón figurasen en el mismo listado que piano, guitarra, violín, flauta u órgano, cuando es evidente que unas y otras palabras no pueden ser definidas conforme a los mismos criterios. Lo mismo sucede con telera, ovejera, estiércol o abono respecto de arado, trillo, bocino o azadón.

\section{LOS NOMBRES DE INSTRUMENTOS AGRÍCOLAS EN EL COSER Y EN EL CORPES XXI}

Una vez que se ha determinado que la lingüística teórica es una fuente de la que puede y debe nutrirse la lexicografía y que aporta información sustancial respecto de los nombres de instrumentos, nos proponemos comprobar en qué medida los corpus, que reflejan 
los usos que hacen los hablantes de las unidades léxicas y de todo tipo de construcciones, confirman o no los datos presentados hasta el momento. Para ello, nos centraremos en los nombres de instrumentos agrícolas ${ }^{23}$, grupo de sustantivos que comparten el hecho de servir para labrar o cultivar la tierra.

Generalmente se señala que los corpus pueden ser empleados para extraer una multitud de datos a propósito del léxico, como los relativos a la frecuencia de uso, al alcance geográfico, al registro, a la cronología, a las combinaciones sintácticas, al ámbito de utilización o al establecimiento de acepciones y su ejemplificación ${ }^{24}$. Sin embargo, los corpus también pueden servir para recabar información acerca de los referentes (en el caso de que las palabras en cuestión los posean) y sobre los rasgos semánticos que no deben faltar en las definiciones de determinados grupos de palabras. Este último aspecto es el que nos interesa, pues nos permite comprobar si los datos proporcionados por la lingüística teórica, a propósito de determinadas construcciones o unidades léxicas (en nuestro caso, los sustantivos que designan instrumentos agrícolas), tienen reflejo en el uso, y también cuáles de ellos resultan más significativos. Las conclusiones extraídas habrán de tener reflejo en las definiciones.

Los corpus que hemos utilizado para nuestra investigación son dos: el Corpus Oral y Sonoro del Español Rural (COSER) y el CORPES XXI. Dirigido por Inés Fernández-Ordóñez, el primero "está formado por grabaciones de la lengua hablada en enclaves rurales de la Península Ibérica”25. Dichas grabaciones se han obtenido a partir de entrevistas semidirigidas realizadas a personas con una edad media de 73 años y con escasa instrucción, en las que se les pregunta sobre cuestiones relacionadas con la vida tradicional del campo. Este es un aspecto de gran interés para nosotros, pues uno de los temas tratados es el de la agricultura. Además, el hecho

23 Concretamente nos hemos centrado en arado, azada, azadón, bielda, bieldo, garia, gario, grada, guadaña, hocino, hoz, rastra, rastrillo, rastro, trilla y trillo.

24 Véase Rojo (2009) para obtener más información al respecto.

25 Tomado de http://www.corpusrural.es/index.php (Consultado el 15 de junio de 2020). 
de que sea únicamente oral aporta un valor diferencial respecto de otros corpus.

Con respecto al segundo, está "formado por textos escritos y orales procedentes de España, América, Filipinas y Guinea Ecuatorial con una distribución de 25 millones de formas por cada uno de los años comprendidos en el periodo 2001 a $2012^{\text {"26 }}$ y de su construcción se ha encargado la Real Academia Española. Se trata de un corpus general (llamado de referencia) que "tiene como propósito básico el de servir para obtener las características globales que presenta una lengua en un momento determinado de su historia" ${ }^{27}$.

El primer corpus nos interesa, obviamente, por su conexión con el léxico de la agricultura, y el segundo, porque se trata de un corpus mucho más amplio, de referencia y que recubre todas las áreas de habla hispana.

Pues bien, la consulta del COSER nos permite comprobar que los informantes dan una gran importancia en sus alocuciones a la función de los instrumentos agrícolas, muy por encima de otras consideraciones $^{28}$ :

(1) Ellas cogían las patatas que yo | bueno es que yo no cogía, yo me encargaba de sacarlas, había que sacarlas con una azada. Enclave: Humada, Burgos, COSER-0922_01).

(2) Les daba de comer to lo que querían y no los obligaba. Aquí había uno, ese que le..., que era un buco, que se los iba..., se los llevaba a cavá con una azá. Enclave: Campos del Río, Murcia, COSER-3104_01.

(3) Si tú no lo arreglas, pos esa..., ese pasto que hay así, la tierra pos lo..., le..., los palos se quemarían y se quedaría eso hecha como dijéramos esto y la ceniza quedaría aquí, pero eso se le iba dando con la aza-, con una azá, íbamos cavando [HS:E2: ¡Ajá!] y se iba quedando abajo. Enclave: Alanís, Sevilla, COSER-3809_01.

\footnotetext{
26 Tomado de https://tinyurl.com/y39jt53c (Consultado el 15 de junio de 2020).

27 Tomado de https://tinyurl.com/y39jt53c (Consultado el 15 de junio de 2020).

28 En los ejemplos del COSER debe interpretarse $I$ como informante y $E$ como entrevistador. Por otro lado, en los ejemplos resaltamos en cursiva, de aquí en adelante, los nombres de instrumentos agrícolas sobre los que nos interesa llamar la atención.
} 
(4) Y entonces, tienes que cavar y dejar un trozo, pues, ¿qué te voy a decir? Una zona así, pero tiene que ser liso, tienes que | con la azada tienes que alisar todo eso, irte al monte y alisar. Enclave: Aulesti, Vizcaya, COSER-4501_01.

(5) E1: Y, ¿cómo, cómo siegan eso con, con guadaña o ya con tractor o...?

I1: Se siega con guadaña y se siega con segadoras. [HS:E1 Y con segadoras.]. Enclave: El Portillo (Solórzano), Cantabria, COSER-1219_01.

(6) I1: ¡Puf! [OTRAS-EM] Pues, primero cortar con la guadaña, que ya estoy yo cortando con el padre y con el hermano, todos, los tres igual. Enclave: Zarimutz (Ekzoriatza), Guipúzcoa, COSER-2012_01.

(7) I2: Yo he trabajao de to. He segao con una hoz, he trillao, [HS:E2 [Asent].] he... lo he hecho to. He trabajao, he arrancao, he... segao. To. Enclave: Aulago (Gérgal), Almería, COSER-0403_01.

Tanto es así que incluso hay un ejemplo en el que el informante, ante la pregunta del entrevistador acerca de cómo es una hoz, contesta explicando cuál es su función:

(8) E1: ¿Y cómo, cómo era esa hoz?

I1: Pues como es ahora: para segar alfalfa y hierba y... lo que sea.

E1: Pero, ¿pequeña, grande...? Enclave: Puebla de Arenoso, Castellón, COSER-1310_01.

Sea como fuere, los informantes también aluden a otros aspectos (ejemplos 9 y 10), en la mayor parte de los casos combinados con la función (ejemplos 11-24):

(9) Denominación alternativa: Ssr: Mira, mira, mira, mira, mira...] Yo os voy a decir una cosa: eh..., los danzantes bailan unos, unos bailes muy bonitos, ¿sabes?; bailan pues..., este año queremos hacer dos que después de la, la pasada guerra civil no se han hecho, que es, eh, los morteros y el del arado o aladro, el del aladro. Enclave: Alcalá de la Selva, Teruel, COSER-4102_02.

(10) Descripción: I: Los trillos... era un..., eran de madera... un trozo así muy grande, más grande que esto y..., y luego lo 
que se enganchaba. Y el trillo tenía debajo, lo que tocaba la tierra, piedrecitas en-, engarzadas en la madera. Enclave: Jérica, Castellón, COSER-1307_01.

(11) Agente + FUnCión: Los gañanes... ¿Sabes cómo...?, ¿quién son los gañanes? [HS:E1: No.] Tampoco, ¿no? Los gañanes son los que cuidan..., los que llevan las..., las yuntas que llevan el arao arando, arando la tierra, son los gañanes. Enclave: Álora, Málaga, COSER-3004_01).

(12) DesCRIPCIÓN + FUnCión: I1: Pues porque se limpiaba más la cepa, [HS:E1 [Asent].] se podía meter en mejores sitios. La hoz, era una cosa que tenía un mango, una cosa así, [G-Mst] por aquí bajo parecía una cuchilla de, de, de un carnicero, de esas pequeñas, [HS:E1 [Asent].] llevaba un corte buenísimo, finísimo y..., y era muy difícil podar, había quien, había que tener su, su aire y su gracia y se cogía un sarmiento así de grande [G-Mst] [HS:E1 [Asent].] y había quien hacía así y le cortaba, [HS:E1 [Asent].] y había que cortar el sarmiento sin que se rajara. Enclave: Valdilecha, Madrid, COSER-2915_01.

(13) Descripción + Función: I1: Con un hocino. Se llama hocino, [HS:I2 Con un hocino.] una cosa que se llama hocino, una cosa | un rabo así, con un yerro de corte que hacía así, y lo segaban. Enclave: Campo Lugar, Cáceres, COSER-1023_01.

(14) Material + FUnCión: Un arado que lo hacía el herrero y el hombre iba en la caballería, lo enganchaban en un apero, un, un arado que se dice y nada y a recoger las almendras y las algarrobas y en las caballerías llevarlas a casa. Enclave: Busot, Alicante, COSER-0304_01.

(15) Función + Agente: El trigo se segaban los hombres. Los hombres lo segaban con un hocino que tenían, y lo segaban. Y luego tenían que sacarlo, bueno. Enclave: Campo Lugar, Cáceres, COSER-1023_01.

(16) Función + Agente: Con una hoz lo segaban, venían gallegos, venían castellanos y lo segaban con hoces. Ahora ya son las maquinarias que hay, [HS:E1 [Asent].] pero antes era con hoces, las hoces. Enclave: Humanes de Madrid, Madrid, COSER-2907_01. 
(17) Función + MOdo De funcionamiento: Claro. Entonces no había tractores. Pos con mulas, con un arao a arado pos a labrar y, y con una azá a excavar. Enclave: Puebla de Don Fadrique, Granada, COSER-1832_01.

(18) FunCión + MOdo DE FUnCIONAMIENTO: I2: No, no, para aquí vale ya... Valía así. Sí. Y luego pues le echabas mineral, echabas mineral y luego cuando venía a segar, pues segarlo. Lo segabas, lo | que antes había que trillarlo a... con yeguas, con un trillo, con yeguas. Enclave: Quintana de los Prados (Espinosa), Burgos, COSER-0939_01.

(19) Modo de Funcionamiento + Función: I1: Pos, pos un par de bestiae, dos muloe, se ponía el..., en asín en el cuello, un ese, se enganchaba en el arao arado y a labrar. Enclave: Doña Inés (Lorca), Murcia, COSER-3106_01.

(20) FUNCIÓN + HIPERÓNIMO + MODO DE FUNCIONAMIENTO:

(21) E1: [Asent]. ¿Pero qué estaba en alto [HS:I Sí, sí, sí.] o...? ¿Y para subir la paja arriba? I: Ah, pues había que... había unas herramientas que se llamaban [R-Ind] bieldos, o garios, [HS:E1 [Asent].] se cogía... con el gario, o con el bieldo, y por un bocín, [HS:E1 [Asent].] como si dijéramos, por una ventana, [HS:E1 [Asent].] uno echándolo y otro a-, a-, adentro [PS] eh... retirándolo para... que c-, cupiera o cu- $\mid$ o... ¿Cómo se dice? Enclave: Muñoveros, Segovia, COSER-3707_01.

(22) Hiperónimo + FUnCión + MOdo DE FUnCiONAMiento: I2: Cuando no, cuando la herramienta esa que tenemos, pos vas volcando el caballón y sacando las patatas, pero a brazo.

E1: ¿Con una azada?

I2: Una azada, a brazo. Sí.

Enclave: Palacios Blancos (Lorquí), Murcia, COSER-3111_01.

(23) FUnCIÓN + DESCRIPCIÓN + SINÓNIMO: I: El trillo po un... lu-, luego había una máquina también de... con unos rodillos de, de, de hierro, ¿sabes? [HS:E1 [Asent].] que iba cortando la paja, [HS:E1 [Asent].] pero el trillo era un, una co-, una trilla era una cosa de madera. Enclave: Fuente del Pino (Jumilla), Murcia, COSER-3107_01. 
(24) Modo de FunCiOnAmiento + DESCRIPCión + FunCión: I: |T9| Pues el trigo y el centeno, se cogía una pareja de vacas, se le ponía un trillo que es de madera, con piedrecitas por debajo y trillábamos, y teníamos que limpiar a mano. Enclave: Peralejos de Abajo, Salamanca, COSER-3611_02.

(25) ObSOlescencia + MOdo de FUnCiOnAMiento + FunCión: I: Pues nada se ataba el trigo, se hacinaba. Bueno, se acarreaba con el carro. Luego se extendía y dar vueltas con el trillo y las mulas. $\mathrm{Y}$ nosotros en la vida pues ha habido una trascendencia de, de, de, de aquello, y luego ya a los tractores y cosechadoras. En eso estuve yo vente años también arando con el tractor. Enclave: Cubillejo del Sitio (Molina de Aragón), Guadalajara, COSER-1907_01.

También hay ocurrencias en que los informantes realizan descripciones de ciertos instrumentos agrícolas, pero como respuesta a preguntas concretas de los entrevistadores a este respecto:

(26) - E1: Y el arado en sí, ¿qué, qué era? ¿Una, una pieza de...?

I1: De hierro. [HS:E1 De hierro.] De hierro... Metálica Enclave: Puebla de Arenoso, Castellón, COSER-1310_01.

(27) I: Igual, igual, echándoles paja y pienso [R-Inf], luego se [A-PIn: reducían] con un yugo [HS:E [Asent].] y a labrar, con..., con un arao [HS:E Con un arado.] de madera.

E: ¿Sí? ¿y cómo era eso?

I: [LT-Rct: El arado cantaré y de piezas iré formando y de la pasión de Cristo, palabras iré explicando.] [RISA] El arado, pues [R-Mcr] tenía reja, [HS:E [Asent].] tenía esteva [A-Inn] fíjate, un surco [R-Mcr] por ahí con un arao el pobre hombre con la esteva. Enclave: Almajano, Soria, COSER-3901_01.

(28) E2: ¿Y qué, cómo era eso?

I1: Una..., una bielda, una horca, como la queráis llamar, una cosa de madera.

I2: Un palo, redondo, como pa un [A-Inn]. Luego una maderita así, gorda, y en la madera agujeritos con guinchos. Enclave: Villaverde-Mogina, Burgos, COSER-0959_01. 
La consulta del CORPES XXI confirma también la prevalencia del rasgo [función] con bastante diferencia sobre el resto, incluso en aquellos casos en los que este no se manifiesta prototípicamente:

(29) Función: Día llegará, si de este modo proseguimos, en que nuestros hijos, en vez de tomar el arado para labrar la tierra o el hacha que sirva para derribar los montes, se dediquen a lavar y planchar la ropa blanca y hasta cortar y adornar los vestidos para las mujeres... (Benítez Rojo, Antonio: Mujer en traje de batalla. Madrid: Alfaguara, 2001. País: Cuba).

(30) FunCión: La siembra se hacía en surcos y en los entresurcos se desarrollaba pronto la vegetación espontánea. La práctica del aricado consistía en dar labor a esos entresurcos con el arado común dos o tres veces al año (Lacasta, C.; Meco, R.: Manual de Agricultura y Ganadería Ecológica. Madrid: Sociedad Española de Agricultura Ecológica, 2002. País: España).

(31) Función: Dos días después de la prueba en Bilbao, José Luis segaba hierba con la guadaña en su prado frente al caserío (Bas, Juan: La cuenta atrás. Barcelona: Destino, 2004. País: España).

(32) FunCión: Empuñó con fuerza la hoz y reanudó la siega interrumpida (Gutiérrez Aragón, Manuel: El ojo del cielo. Barcelona: Anagrama, 2018. País: España).

(33) FunCión NO PROTOTíPICA: Pero la vaca no se movió, sólo la cola subió y bajó para espantar unas cuantas moscas. La golpeó con la azada en los ijares. Las orejas vibraron, pero el cuerpo seguía clavado en la tierra, firme en su decisión de estar clavado en la tierra, la cabeza gacha, exhalando un fuerte olor a heno y a bosta (Sánchez-Andrade, Cristina: Bueyes y rosas dormían. Madrid: Ediciones Siruela, 2001. País: España).

(34) FunCIÓN NO PROTOTÍPICA: Los dos bandos usaron lanzas, azadas y machetes para matar a todo aquel que identificaban como miembro de la comunidad rival, señalaron las emisoras, que citan testigos presenciales (“AGENCIAS. - KINSHASA”. El Diario Vasco. San Sebastián: Sociedad Vascongada de Publicaciones, 23/01/2003. País: España). 
Se alude asimismo a otros aspectos, algunos de ellos no presentes en el COSER: cualidad, símbolo, metáfora o información enciclopédica. Eso sí, también en algunos de estos casos la función está presente, en combinación con otros rasgos ${ }^{29}$ :

(35) Cualidad: En cuanto estuvieron solos, Nacho fue a abrazarla, pero ella lo detuvo en seco, interponiendo un brazo estirado que parecía una barrera de guardarrafles tan afilados como una boz (Vallvey, Ángela: Muerte entre poetas. Barcelona: Planeta, 2008. País: España).

(36) Elementos compositivos: Agarró el palo del rastrillo y se lo quitó de las manos (Gutiérrez Aragón, Manuel: El ojo del cielo. Barcelona: Anagrama, 2018. País: España).

(37) Hiperónimo: Las herramientas necesarias para hacerla son una trituradora, jimador, bieldo, pala recta y curva, cubeta o manguera, plástico obscuro para cubrir y un arnero para el terminado final (Oliver Guadarrama, Rogelio: “iManos a la composta!”. Hypatia. Morelos: hypatia.morelos.gob.mx, 07/2007. País: México).

(38) Modo de funcionamiento: Los más significativos son los clareos y claras, consistentes en la reducción de la densidad del arbolado eliminando los no deseados para obtener espesuras acordes con su desarrollo; la poda, es decir, la eliminación por corta de las ramas con el fin de conformar fustes y masas más adecuadas al objetivo de gestión establecido, y el desbroce, bien sea manual (azada, hacha) o mecánico (motodesbrozadora), que sirve para eliminar el matorral mediante operaciones de arranque, descuaje o roza (Fernández, T.: "Medio Ambiente invierte 40 millones en tratamientos silvícolas preventivos". Diario de León.es. León: diariodeleon.es, 20/07/2004. País: España).

(39) INFORMACIÓN ENCICLOPÉDICA: La ocupación romana supuso un gran aporte en la difusión de la cultura mediterránea, basada

29 Algunos de los lemas a los que nos estamos refiriendo han desarrollado acepciones propias en diversos países hispanoamericanos, tal y como puede comprobarse en el Diccionario de americanismos de la ASALE (2010), a propósito de arado, azadón, grada, rastra, rastrillo, rastro, trilla y trillo. 
principalmente en el trigo, el pan y el aceite; mejoraron los sistemas de cultivo con el arado y primitivos sistemas de regadío (Armendáriz Sanz, José Luis: Procesos de cocina. Madrid: Paraninfo, 2001. País: España).

(40) Símbolo: Ahí van también un hombre lobo, un vampiro, varias brujas y calaveras con sus inseparables guadañas que representan a la muerte (Urías, Tania: "Las calabiuzas. Fiesta de la mitología cuscatleca”. El Diario de Hoy. Hablemos on line. San Salvador: elsalvador.com, 14/11/2004. País: El Salvador).

(41) Símbolo: Tres elementos superpuestos configuran sus imágenes: el sol, primero y al fondo, con una luminosidad radiante a través de fuertes rayos que se expanden sin límites; luego, tensa hacia la izquierda, la bandera comunista, con la boz campesina cruzándose con el martillo obrero; finalmente, y en el plano más cercano a nosotros, el clásico retrato de Abimael Guzmán con esos lentes tan gruesos que le ocupan casi una tercera parte de la cara (Vich, Víctor: El caníbal es el Otro. Violencia y cultura en el Perú contemporáneo. Lima: IEP Ediciones, 2002. País: Perú).

(42) AgENTE + FUNCIÓN: Los europeos, en cambio, labraban la tierra con sus arados y sembraban al voleo grandes extensiones de un solo cultivo que cosechaban con guadañas (Aguilar, Jasmín; Illsley, Catarina; Marielle, Catherine: "Los sistemas agrícolas de maíz y sus procesos técnicos”. Esteva, Gustavo; Marielle, Catherine [coords.]: Sin maíz no hay país. Ciudad de México: Museo Nacional de Culturas Populares, 2003. País: México).

(43) DeSCRIPCIÓN + FUnCIÓN: Mi madre empuñaba una especie de escoba de metal con la que rastrillaba las hojas antes de depositarlas en una carretilla, donde ya había un montón de abrojos y zarzas extraídas de raíz. Busqué otro rastrillo y recogí algunas hojas, y mientras lo hacía, sin levantar la vista del suelo, se lo conté todo, sin dejarme nada en el tintero (Rivera de la Cruz, Marta: En tiempo de prodigios. Barcelona: Planeta, 2006. País: España).

(44) FunCión + AGENTE: En general, en esta zona se tiende al paso de la agricultura de subsistencia hacia cultivos comerciales (chicozapote, mamey, mango, cítricos, ajonjolí, algodón, sorgo) 
y la cría de ganado. Diversos grupos indígenas y mestizos han adoptado el uso de tractores, rastras y arados, sobre todo en las llanuras costeras, para cultivos comerciales (Aguilar, Jasmín; Illsley, Catarina; Marielle, Catherine: "Los sistemas agrícolas de maíz y sus procesos técnicos”. Esteva, Gustavo; Marielle, Catherine [coords.]: Sin maíz no hay país. Ciudad de México: Museo Nacional de Culturas Populares, 2003. País: México).

(45) Metáfora + FUnCIÓn: El sida es la gran hoz que lo cercena todo; ahora se añade el hambre (Bayón Pereda, Miguel: "La muerte acecha a África”. El País. Madrid: elpais.com, 12/09/2002. País: España).

(46) Hiperónimo + Ámbito de uso: Un rastrillo fabricado en la provincia se presenta en la muestra de maquinaria agrícola ("MAVE. La XVII Demostración de Recolectores de Forraje que se celebra mañana reunirá ocho máquinas". El Norte de Castilla. Valladolid: nortedecastilla.es, 21/06/2001. País: España).

(47) INFORMACIÓN ENCICLOPÉDICA + SÍMBOLO: Otro invento medieval es la guadaña. No es extraño que el símbolo de la muerte sea un esqueleto con una guadaña, ya que las pestes negras también son propias del Medievo. La guadaña era el iPhone de la época, por lo tanto, la lógica es que a la muerte la representaran dotada de la más avanzada tecnología (Mulet, José Miguel: Transgénicos sin miedo. Barcelona: Planeta, 2017. País: España).

(48) Hiperónimo + material: En cuanto a las fuerzas productivas, la tecnología empleada en la parcela del encomendero resultó de una fusión de elementos europeos e indígenas: a la técnica de trabajo de los indígenas Guaraní se incorporaron como nuevos medios de trabajo, herramientas de metal — hacha, azada y arado- y tracción animal, y a los cultivos indígenas se sumaron la caña de azúcar, la naranja y el banano (Fogel, Ramón: Pobreza y rol del Estado en el Paraguay. Asunción: Centro de Estudios Rurales Interdisciplinarios, 2002. País: Paraguay).

Tanto el COSER como el CORPES XXI coinciden, pues, en conceder un protagonismo claro a la función, en línea con los postulados establecidos por la lingüística teórica a propósito de los 
nombres de instrumentos; incluso lo que hemos catalogado como modo de funcionamiento presupone, en realidad, la existencia de una función. El segundo presenta, no obstante, una casuística un tanto más variada, como cabe esperar de un corpus de referencia que contiene, además, 25 millones de formas incluidas en textos de variado tipo; de ahí las alusiones enciclopédicas o simbólicas que no tienen presencia en el COSER. No debe olvidarse que en este último los nombres de instrumentos agrícolas se mencionan en entrevistas semidirigidas en las que se les pregunta a los informantes acerca de cuestiones relacionadas con la vida del campo, lo cual acarrea que la información proporcionada resulte más prototípica.

También es importante señalar que no todas las propiedades que comparten los nombres de instrumentos y de las que informa la lingüística teórica parecen tener similar reflejo en el uso. Así, en páginas anteriores se explicaba que los nombres de objetos fabricados son agentivos y admiten complementos de materia y adjetivos relacionados con su buen o mal funcionamiento o con cualidades relacionadas con su función. Sin embargo, el rasgo [agente] no es equiparable, en términos cuantitativos, a la función, ni tampoco el rasgo [cualidad], del que tan solo hemos encontrado un caso en uno de los corpus (el CORPES XXI). Con respecto al material, también se halla muy lejos de la importancia concedida a la función, si bien es cierto que las alusiones al material, elementos compositivos o descripción en general alcanzan mayor presencia. Ello puede deberse a los siguientes factores:

1. En el COSER los informantes contestan a preguntas de los entrevistadores, quienes en no pocas ocasiones se interesan por cómo son los instrumentos agrícolas a los que aquellos se están refiriendo (ejemplos 25-27).

2. Algunos de estos instrumentos se identifican con épocas pasadas o, al menos, con el medio rural. Por ello los hablantes, especialmente los informantes del COSER, quienes son personas de avanzada edad, pueden sentir la necesidad de referirse a algunos de estos instrumentos agrícolas haciendo 
ciertas aclaraciones, sobre todo ante entrevistadores más jóvenes y no residentes en sus respectivas localidades ${ }^{30}$.

3. No hay muchas diferencias entre algunos de los instrumentos en cuestión, por lo que la descripción podría resultar un aspecto clave para su identificación. Nótense en este sentido las definiciones de azada y azadón incluidas en el DLE de la RAE (el subrayado es nuestro):

azada 1. f. Instrumento que consiste en una lámina o pala cuadrangular de hierro, ordinariamente de 20 a $25 \mathrm{~cm}$ de lado, cortante uno de estos y provisto el opuesto de un anillo donde encaja y se sujeta el astil o mango, formando con la pala un ángulo un tanto agudo. Sirve para cavar tierras roturadas o blandas, remover el estiércol, amasar la cal para mortero, etc.

azadón 1. m. Instrumento que se distingue de la azada en que la pala, cuadrangular, es algo curva y más larga que ancha, y que sirve para rozar y romper tierras duras, cortar raíces delgadas y otros usos análogos.

Esto convierte el rasgo [descripción], al menos en casos como este, en diferencia específica en una definición de tipo hiperonímica. Y el rasgo [hiperónimo] (en este caso, instrumento), como es lógico, constituye el primer elemento delimitador de la definición. No puede olvidarse que, si se parte de una concepción relacional del léxico como la que se propugna en estas páginas, a través de la hiperonimia cabe relacionar no pocas palabras. En este sentido, parece aconsejable apuntar, en la medida de lo posible, hacia un modelo definitorio en el que el hiperónimo encabece la definición y a este le siga la función. No cabe duda de que la descripción debe estar presente también en las definiciones de instrumentos muy próximos (caso de azada y azadón o de bielda y bieldo), si bien no

\footnotetext{
30 El siguiente ejemplo ilustra perfectamente esta situación (la negrita es nuestra): I1: Con unas ruedas, y pues con aquel carro y las vacas que teníamos pues traíamos el pan, pa, pa la era, lo cargábamos el carro y lo traíamos pa la era después de que ya lo teníamos en la era pues ya lo trillábamos con un trillo, como nunca haís oído nada de eso ¡qué vais a saber!, teníamos unos trillos, que trillábamos el pan. ¡Ah, mira la hoz! Enclave: Lucillo, León, COSER-2644_01.
} 
sería descabellado optar por incluir este rasgo en la definición de otros lemas o incluso en el resto de lemas que nos conciernen. Esta forma de actuación es adecuada si consideramos que, ante sustantivos con referentes un tanto desconocidos por no pocas personas en la sociedad actual, la inclusión de ese tipo de información puede resultar esclarecedora. En nuestra opinión, lo importante es que a la toma de decisiones subyazca un criterio claro que justifique la adopción de las mismas, y que ello tenga como resultado un producto sistemático. Consideramos, pues, una propuesta plausible la de incluir en las definiciones los tres rasgos mencionados y hacerlo a partir de un mismo orden, siempre y cuando no haya otros factores que interfieran.

En cualquier caso, queda claro que la lingüística teórica proporciona información valiosísima para la elaboración de los diccionarios, pero no todos los datos recabados por aquella son igualmente importantes para la lexicografía ni aplicables en la misma medida. La tarea del lexicógrafo no puede limitarse al volcado de los datos de una a otra disciplina, sino que este ha de seleccionar los más relevantes en función de los objetivos perseguidos e indagar cuáles se ajustan mejor a las técnicas lexicográficas y qué uso hacen los hablantes de determinado grupo de palabras. En esta compleja tarea los corpus son fundamentales, y no solo en las funciones que habitualmente les vienen siendo asignadas, sino también en la selección de rasgos definitorios que han de figurar en las definiciones y en la conformación, por tanto, de modelos definitorios.

\section{Conclusiones}

En las páginas precedentes se ha abogado por relacionar de manera estrecha la lingüística teórica y la lexicografía como fórmula metodológica para mejorar los diccionarios tanto en sus etapas de planificación como de redacción. Consideramos probado que ello mejora sustancialmente el producto final, que gana en solidez, coherencia interna, rigor y precisión. Entablar ese tipo de conexión no siempre es sencillo, si bien genera resultados muy interesantes en 
lo relativo a la parcelación del léxico y a la aplicación de modelos de definición a grupos de palabras más o menos homogéneos.

El modo de llevar a cabo estas premisas, en cualquier caso, no es tarea fácil, sino que requiere de un complejo proceso en el que el lexicógrafo ha de determinar qué información proporcionada por la lingüística teórica es la que desempeña un papel más determinante en la descripción de un tipo concreto de léxico en un diccionario. Una manera de conseguirlo puede ser a través de la consulta de corpus, lo cual supone anclar esa información en datos relacionados estrictamente con el uso que los hablantes dan a una unidad léxica o construcción concretas. En este sentido, la colaboración entre la lingüística de corpus y la lexicografía abre un camino muy fructífero para el avance en la construcción de los diccionarios. No nos referimos solo a la utilización de los corpus como recursos para la obtención de ejemplos, el establecimiento de acepciones, para constatar el alcance geográfico de una determinada forma, etc., sino como medios para determinar qué tipo de información puede resultar más relevante a la hora de caracterizar semánticamente un determinado lema.

Nosotros nos hemos centrado en un conjunto de palabras (los nombres que designan instrumentos agrícolas), en cuyas definiciones no ha de faltar el hiperónimo, la función y, al menos en ciertas ocasiones, la descripción. Asimismo, sería muy importante optar por un vocabulario definidor en la redacción de dichas definiciones, porque ello no solo garantizaría que se codificara de manera homogénea y sistemática un determinado grupo de lemas, sino también que se incluyera en ellas igual o parecido tipo de información, en la medida en que esto sea posible.

\section{REFERENCIAS BIBLIOGRÁFICAS}

Apresjan, Juri

2000 Systematic lexicography. New York: Oxford University Press.

Asociación de Academias de la Lengua Española (Asale)

2010 Diccionario de americanismos. Madrid: Santillana. Consultado: junio de 2020. <http://lema.rae.es/damer>. 
AtKins, B. T. Sue y Michael Rundell

2008 Practical Lexicography. Oxford: Oxford University Press.

Barrios Rodríguez, María Auxiliadora y Elena De Miguel Aparicio

2018 "Finalidad, función y telicidad: la información télica y su relación con artefactos, instrumentos y objetos auxiliares”. Círculo de Lingüística Aplicada a la Comunicación (CLAC). 75, 125-154. https://doi.org/10.5209/CLAC.61351

Battaner, María Paz

2014 "El diccionario como punto de encuentro en la descripción de la lengua española”. Ponencia presentada en el Simposio Internacional El futuro de los diccionarios en la era digital. Grabación disponible en https://tinyurl.com/y4pw9llw.

Batiukova, Olga

2009 "Aplicaciones lexicográficas de la teoría del Lexicón Generativo". En Fronteras de un diccionario. Las palabras en movimiento. Eds., Elena de Miguel, Santiago U. Sánchez Jiménez, Ana Serradilla Castaño, Romana-Anca Radulescu y Olga atiukova. San Millán de la Cogolla: Cilengua, 231-268.

Bosque, Ignacio

1982 "Sobre la teoría de la definición lexicográfica". Verba. 9, 105123.

Bosque, Ignacio

1999 "El nombre común”. En Gramática descriptiva de la lengua española. Dirs., Ignacio Bosque y Violeta Demonte. Madrid: Espasa, 3-75.

Bosque, Ignacio

2014 "Ideas para conectar palabras". Ponencia presentada en el Simposio Internacional El futuro de los diccionarios en la era digital. Grabación disponible en https://tinyurl.com/y5pnaq7g.

Campos Souto, Mar

2015 "El NDHE como muestra de la nueva lexicografía digital”. Estudios de Lexicografía (monográfico sobre el Nuevo Diccionario Histórico del Español. Dir., José Antonio Pascual). 3, 71-93.

Decesaris, Janet y Elisenda Bernal

2005 "La gramática de los nombres de materia en los diccionarios”. En Entre la semántica léxica, teoría del léxico y sintaxis. 
Coords., Juan Cuartero Otal y Gerd Wotjak. Frankfurt am Main: Peter Lang, 453-465.

FERNÁNDEZ-ORdÓÑEZ, Inés (dir.)

2005 Corpus Oral y Sonoro del Español Rural. Consultado: junio de 2020. $<$ www.corpusrural.es $>$.

Flaux, Nelly y Danièle van de Velde

2000

Les noms en français: esquisse de classement. Paris:

Ophrys.

García Meseguer, Álvaro

2008 Clases y categorías de nombres comunes: un nuevo enfoque. Madrid: Arco/Libros.

GonZÁlez Cobas, Jacinto

2009 "Los instrumentos de medida en el DRAE: una propuesta de modelo de definición”. En Fronteras de un diccionario. Las palabras en movimiento. Eds., Elena de Miguel, Santiago U. Sánchez Jiménez, Ana Serradilla Castaño, Romana-Anca Radulescu y Olga Batiukova. San Millán de la Cogolla: Cilengua, 121-147.

GonZÁlez CoBAs, Jacinto

2010 "Caracterización lingüística de los nombres de instrumentos de medida. Repercusiones lexicográficas”. Revista de Lexicografía. XVI, 39-58.

GonZález Cobas, Jacinto

2011 "Cómo definir los nombres de instrumentos musicales. Propuesta lexicográfica”. Revista de Lexicografía. XVII, 65-82.

GonzÁlez Cobas, Jacinto

2016 "Lexicografía y sistematicidad. Acerca de los nombres que designan instrumentos agrícolas”. Revista de Lexicografía. XXII, 17-30.

Gross, Gaston

1998 “Introduction aux classes d'objets”. Langages. 131, 6-33.

Haensch, Günther, Lothar Wolf, Stefan EtTinger y Reinhold Werner

1982 La lexicografía. De la lingüistica teórica a la lexicografía práctica. Madrid: Gredos. 
Herrero Ingelmo, José Luis

2004 "El diccionario electrónico: palabras que son oraciones (de los aplausos a los abucheos)". En De lexicografía: Actas del I Simposium Internacional de Lexicografía. Coords., María Paz Battaner y Janet Ann DeCesaris. Barcelona: Universitat Pompeu Fabra, 451-460.

Maldonado GonzÁlez, Concepción

2012 "Los diccionarios en el mundo ELE: ayer, hoy y mañana (una reflexión desde la propia experiencia)". Revista Internacional de Lenguas Extranjeras. 1, 151-179.

Menéndez Pidal, Ramón

1953 "Prólogo". En Vox. Diccionario general ilustrado de la lengua española. Segunda ed. corregida y notablemente ampliada por Samuel Gili Gaya. Barcelona: Ediciones Spes.

Pascual, José Antonio

1996 "La coherencia en los diccionarios de uso". En Léxico y diccionarios. Coord., Esther Forgas. Tarragona: Departament de Filologies Romàniques, Universitat Rovira i Virgili, 167-198.

Pascual, José Antonio

2015 "Introducción a una celebración lexicográfica: a propósito de la reciente publicación de un millar de palabras del NDHE en el portal de la RAE". Estudios de Lexicografía (monográfico sobre el Nuevo Diccionario Histórico del Español. Dir., José Antonio Pascual). 3, 7-13.

Pustejovsky, James

1995 The Generative Lexicon. Cambridge: MIT Press.

Pustejovsky, James y Olga Batiukova

2019 The Lexicon. Cambridge: Cambridge University Press.

Real Academia Española

$\mathrm{s} / \mathrm{f} \quad$ Corpus del Español del Siglo XXI (CORPES). Banco de datos (CORPES XXI) [en línea]. Consultado: junio de 2020. <http:// www.rae.es>.

Real Academia Española

2013 Diccionario bistórico de la lengua española (DHLE) [en línea]. Consultado: junio de 2020. <http://web.frl.es/DH>. 
Real Academia Española

2014 Diccionario de la lengua española. [Actualización en línea 2019]. Madrid: Espasa. Consultado: junio de 2020. <https:// dle.rae.es>.

Real Academia Española (RAE) y Asociación de Academias de la LENGUA EsPañola (ASALE)

2009 Nueva gramática de la lengua española. Madrid: Espasa.

Rojo, Guillermo

2009 "Sobre la construcción de diccionarios basados en corpus". Revista Tradumática - Traducció $i$ Tecnologies de la Informació i la Comunicació. 7, 1-7.

Salas Quesada, Pilar y Abelardo Torres Morcillo

2015 "Aproximación a los fundamentos del NDHE a través de las herramientas informáticas usadas en su elaboración y presentación”. Estudios de Lexicografía (monográfico sobre el Nuevo Diccionario Histórico del Español. Dir. José Antonio Pascual). 3, 15-69.

SOler i Bou, Joan

2014 Intervención en la mesa redonda Lexicografía y lingüística, dentro del Simposio Internacional El futuro de los diccionarios en la era digital. Grabación disponible en https://tinyurl.com/ y32vgucz. 